\title{
Efeitos da sondagem nasogástrica em pacientes com acidente cerebrovascular e disfagia
}

\author{
Effects of nasogastric catheterization in patients with stroke and dysphagia \\ Efectos de la cateterización nasogástrica en pacientes con accidente cerebrovascular y disfagia
}

\section{Tahissa Frota Cavalcante', Thelma Leite de Araújo", Ana Railka de Souza Oliveira"II}

' Universidade da Integração Internacional da Lusofonia Afro-Brasileira, Curso de Enfermagem. Redenção-CE, Brasil.

"Universidade Federal do Ceará, Departamento de Enfermagem,

Programa de Pós-Graduação em Enfermagem. Fortaleza-CE, Brasil.

"I' Universidade de São Paulo, Escola de Enfermagem, Programa de Pós-Graduação em Enfermagem. Ribeirão Preto-SP, Brasil.

\section{Submissão: 25-04-2013 Aprovação: 11-08-2014}

\section{RESUMO}

O estudo teve por objetivo analisar os efeitos da sondagem gástrica em pacientes com acidente vascular cerebral e disfagia. Revisão sistemática da literatura, realizada em seis bases de dados, com os descritores stroke e intubation, gastrointestinal. Foram encontrados 120 estudos e selecionados três ensaios clínicos. Os resultados apontaram diferentes desfechos, entre os quais: aumento do nível sérico de albumina (gastrostomia), prognóstico ruim e risco de morte (gastrostomia), aumento das falhas no tratamento devido a bloqueio, deslocamento e reinserção da sonda nasogástrica, e aumento da incidência de hemorragia gastrointestinal (sonda nasogástrica). A partir dos resultados obtidos nesta revisão sistemática, ressaltam-se as seguintes evidências: a sondagem nasogástrica deve ser adotada precocemente como um método de alimentação enteral; as falhas do tratamento são mais comuns naqueles que utilizam a sonda nasogástrica como método de alimentação; os resultados relacionados à melhora do estado funcional dos pacientes foram semelhantes, independente do método de terapia nutricional empregado.

Descritores: Enfermagem; Intubação Gastrointestinal; Acidente Vascular Cerebral.

This study aimed to analyze the effects of gastric intubation in patients with stroke and dysphagia. A systematic literature review was performed in six databases, using the keywords stroke and intubation, gastrointestinal. One hundred and twenty studies were found, from which three clinical trials were selected. The results showed different outcomes, including: increased serum albumin level (gastrostomy), poor prognosis and risk of death (gastrostomy), increased treatment failures because of blocking, displacement and reinsertion need of the nasogastric tube, and increased incidence of gastrointestinal bleeding (nasogastric tube). From the results obtained in this systematic review, we emphasize the following evidences: a nasogastric catheter should be adopted as a method of early enteral feeding; treatment failures are more common in those who use nasogastric tube-feeding; outcomes related to improved functional status of patients were similar, regardless of the method of nutritional therapy used.

Key words: Nursing; Intubation; Gastrointestinal; Stroke.

\section{RESUMEN}

El objetivo del estudio fue analizar los efectos de la intubación gástrica en pacientes con accidente cerebrovascular y disfagia. Se llevó a cabo una revisión sistemática de la literatura en seis bases de datos, utilizando-se las palabras clave accidente cerebrovascular y intubación, gastrointestinal. Entre 120 estudios identificados, fueran seleccionados tres ensayos clínicos. Los resultados mostraron diferentes desfechos, incluyendo: aumento del nivel de albúmina sérica (gastrostomía); mal pronóstico y riesgo de muerte (gastrostomía); aumento de los fracasos del tratamiento debido a obstrucción, desplazamiento y necesidad de reinserción de la sonda nasogástrica; y aumento de la incidencia de hemorragia gastrointestinal (sonda nasogástrica). A partir de los resultados obtenidos, destacamos las siguientes evidencias: se deben adoptar catéteres nasogástricas como método de alimentación enteral temprana; fracasos del tratamiento son más comunes en aquellos que utilizan la alimentación con sonda nasogástrica; los resultados relacionados con la mejora del estado funcional de los pacientes fueron similares, independientemente del método de terapia nutricional utilizado.

Palabras clave: Enfermería; Intubación Gastrointestinal; Acidente Cerebrovascular.

\section{AUTOR CORRESPONDENTE Tahissa Frota Cavalcante E-mail: tahissa@unilab.edu.br}




\section{INTRODUÇÃO}

O estudo tem por objeto a análise dos efeitos da sondagem gástrica em pacientes com acidente vascular cerebral e disfagia. A motivação principal para a realização desta pesquisa surgiu em decorrência dos resultados encontrados em estudo de doutorado relacionado à validação do diagnóstico de enfermagem Risco de aspiração em pacientes com acidente vascular cerebral ${ }^{(1)}$.

De acordo com os resultados deste estudo, o diagnóstico de enfermagem Risco de aspiração foi definido como o risco de entrada de secreções gastrintestinais, secreções orofaríngeas, sólidos ou líquidos abaixo das cordas vocais para o trato respiratório inferior. Os fatores de risco foram relacionados para os pacientes com acidente vascular cerebral: disfagia, reflexo de tosse prejudicado ou ausente, desordens neurológicas, uso de tubos gastrintestinais, mobilidade corporal diminuída, reflexo de vômito diminuído ou ausente e cabeceira do leito baixa ${ }^{(1)}$.

Em relação ao fator de risco uso de tubos gastrintestinais, pela análise da literatura e na prática clínica constatou-se ser um tema bastante controverso, pois é comum encontrar a adoção da sondagem nasogástrica para a nutrição enteral em pacientes com acidente vascular cerebral e disfagia. Este fato pode ser corroborado por outro estudo(2), no qual afirma que a sondagem nasogástrica é um método utilizado para a nutrição enteral e para a prevenção da aspiração respiratória em pacientes com acidente vascular cerebral. É oportuno ressaltar que a sonda nasogástrica é o método de escolha no cuidado agudo nutricional do paciente com acidente vascular cerebral, por ser mais barata e oportunizar a administração de medicamentos por via enteral.

Autores alertam ${ }^{(2-3)}$ que o uso da sonda nasogástrica afeta a deglutição em pessoas saudáveis, pois diminui a habilidade para deglutir e prejudica a elevação laríngea, os quais são mecanismos protetores das vias aéreas. Assim, teoricamente, o risco de aspiração estaria aumentado pelo o uso da sondagem nasogástrica, fato que foi comprovado anteriormente por estudo que acompanhou pacientes disfágicos com $\mathrm{AVC}^{(4)}$. Dessa forma, estudos asseveram ${ }^{(5-6)}$ que muitos clínicos utilizam a alimentação enteral como um método eficaz de nutrição e prevenção da pneumonia aspirativa. Todavia, o risco de aspiração em pacientes com refluxo gastroesofágico é aumentado pela utilização de tubos alimentares.

Não obstante, o uso das sondas nasogástricas em pacientes com acidente vascular cerebral pode reverter os efeitos da má-nutrição daqueles que não são hábeis para se alimentar sozinhos ou os que têm disfagia. Aproximadamente, um terço dos pacientes com acidente vascular cerebral necessita utilizar tubos alimentares para a nutrição enteral na fase aguda da doença e, destes, $10 \%$ dependem desta intervenção até seis meses após o acidente vascular cerebral ${ }^{(7)}$.

Entretanto, há poucos estudos que examinaram a frequência, as indicações do uso da sonda nasogástrica e as complicações em pacientes com acidente vascular cerebral ${ }^{(8)}$. Importa destacar que os diagnósticos de enfermagem de Deglutição prejudicada e Risco de aspiração são frequentemente encontrados em pacientes com acidente vascular cerebral ${ }^{(1)}$.

Em virtude da associação da disfagia com a aspiração respiratória, a má-nutrição e a desidratação, esta apresenta relação com a incidência da pneumonia aspirativa, com o aumento do tempo de internação, da institucionalização e da mortalidade. A pneumonia é uma séria complicação que ocorre nas primeiras 48 a 72 horas após o acidente vascular cerebral e é responsável por aproximadamente $15 \%$ a $25 \%$ das mortes relacionadas a essa doença ${ }^{(9)}$.

O elemento chave no tratamento dos pacientes com acidente vascular cerebral na fase aguda e subaguda está baseado na prevenção de complicações, como: problemas associados à pneumonia aspirativa, hiperglicemia, febre, edema cerebral, desidratação, desnutrição e tromboembolismo ${ }^{(9)}$.

A partir deste contexto, surgiu o seguinte questionamento: Quais são os efeitos da sondagem gástrica em pacientes com acidente vascular cerebral e disfagia? Diante deste questionamento, este estudo propõe realizar uma revisão sistemática sobre os efeitos da sondagem gástrica em pacientes com acidente vascular cerebral e disfagia.

Diversos estudiosos afirmam que o método da revisão sistemática da literatura pode identificar os efeitos benéficos e nocivos de diferentes intervenções da prática assistencial, estabelecer lacunas do conhecimento e identificar áreas que necessitam de futuras pesquisas na Enfermagem, com implicações para a assistência prestada. Ressaltam, ainda, que a revisão sistemática é um recurso valioso de informações para a tomada de decisões ${ }^{(10-11)}$.

Este estudo é relevante por proporcionar uma síntese do conhecimento baseado em evidências científicas sobre a sondagem nasogástrica em pacientes com acidente vascular cerebral disfágicos, facilitando, assim, a atualização do conhecimento e a tomada de decisões dos enfermeiros clínicos para o cuidado de enfermagem destes pacientes.

\section{METODOLOGIA}

Conforme referido, o método de pesquisa adotado para a realização deste estudo foi a revisão sistemática da literatura, uma forma de síntese das informações disponíveis em dado momento, sobre um problema específico, de forma objetiva e reproduzível, por meio de método científico. Tem como princípios gerais a exaustão na busca dos estudos analisados, a seleção justificada dos estudos por critérios de inclusão e exclusão explícitos e a avaliação da qualidade metodológica, bem como a quantificação do efeito dos tratamentos por meio de técnicas estatísticas ${ }^{(10-11)}$.

Foram seguidos os seguintes passos para a realização da revisão sistemática: definição da questão, busca na literatura, seleção dos estudos, avaliação crítica da qualidade metodológica (escore de Jadad), coleta e síntese dos dados e análise dos dados ${ }^{(12)}$.

A questão de pesquisa da revisão sistemática da literatura foi elaborada segundo a estratégia PICO ${ }^{(13)}$ : Quais são os efeitos da sondagem gástrica quando comparados a outros métodos de alimentação em pacientes disfágicos com acidente vascular cerebral? 
Quadro 1 - Estratégia PICO adotada no estudo, Fortaleza-CE, 2012

\begin{tabular}{|c|c|c|c|}
\hline População & Intervenção & Comparação & Resultados \\
\hline $\begin{array}{l}\text { Pessoas com acidente vascular } \\
\text { cerebral, independente do tipo } \\
\text { (isquêmico ou hemorrágico) e } \\
\text { disfágicos. } \\
\text { Pessoas com acidente vascular } \\
\text { cerebral independente da fase } \\
\text { da doença (aguda, subaguda ou } \\
\text { reabilitação) e disfágicos. }\end{array}$ & Uso da sondagem gástrica. & $\begin{array}{l}\text { A comparação pode ser com } \\
\text { nenhum método de alimen- } \\
\text { tação, com outros métodos de } \\
\text { alimentação enteral (sondagem } \\
\text { nasoenteral, gastrostomia ou je- } \\
\text { junostomia). }\end{array}$ & $\begin{array}{l}\text { Proporção de pacientes sem } \\
\text { complicações em relação ao } \\
\text { uso da sondagem nasogástrica } \\
\text { comparada àqueles com com- } \\
\text { plicações. }\end{array}$ \\
\hline
\end{tabular}

A busca nas bases de dados foi realizada por um pesquisador independente e para minimizar possíveis vieses foi selecionado um número maior de artigos primários, que permitisse a busca de evidências para a questão investigada. A seleção dos artigos ocorreu nas seguintes bases de dados: Latin American and Caribean Health Science Literature Database (Lilacs), Pubmed (serviço da USA National Library of Medicine) Cumulative Index to Nursing and Allied Health Literature (Cinahl), Scopus, Web of Science e Cochrane Central Register of Controlled Trials (CENTRAL, Clinical Trials).

A estratégia de busca considerou o conjunto de termos relacionados à população do estudo $(\mathrm{P})$ e à intervenção avaliada (I). Logo, foram selecionados descritores dos vocabulários controlados de cada base de dados (DeCS DATABASE, MeSH DATABASE e títulos CINAHL), os quais foram: Stroke (P) and Intubation, Gastrointestinal (I).

Os critérios de inclusão estabelecidos para os estudos foram: a) estudos de ensaios clínicos controlados e randomizados; b) disponíveis nos idiomas português, inglês ou espanhol; c) completos que abordem a temática da pesquisa e que respondam à questão de pesquisa. Como critérios de exclusão foram adotadas as recomendações de um estudo ${ }^{(12)}$, as quais preconizam que para a realização de uma revisão sistemática, os estudos de revisão narrativa, revisão integrativa, relatos de casos ou comunicações breves, bem como, estudos de baixa qualidade ou que apresentem erros sistemáticos devem ser excluídos.

A busca bibliográfica foi realizada no período de dezembro de 2012 a fevereiro de 2013. Cada base de dados acessada foi esgotada em um único dia, com gravação da página de busca. A seleção dos estudos foi realizada nos dias subsequentes.
Ressalta-se que, para estabelecer de forma adequada os critérios de inclusão e exclusão, primeiramente eram lidos o título e o resumo do estudo. Quando ambos não proporcionaram segurança ao pesquisador, foi acessado o texto completo e realizada leitura flutuante para empregar adequadamente os critérios de inclusão e exclusão.

Os trabalhos incluídos inicialmente foram acessados na íntegra para a avaliação da qualidade metodológica, de acordo com uma escala específica ${ }^{(14)}$, que avalia os seguintes aspectos: 1) o estudo foi descrito como aleatório? 2) o método de randomização foi apropriado? 3) o estudo foi descrito como duplo-cego? 4) o método de mascaramento usado foi apropriado? 5) houve descrição de exclusões e perdas?

Esta escala ${ }^{(14)}$ concede de um a cinco pontos para os ensaios clínicos randômicos. Aqueles com um a dois pontos são considerados de baixa qualidade e aqueles com três a cinco pontos são considerados de alta qualidade ${ }^{(15)}$.

Destaca-se que a avaliação da qualidade metodológica foi realizada por dois pesquisadores. Conforme referido apenas àqueles estudos que atenderem a todos os critérios de inclusão e exclusão estabelecidos fizeram parte da amostra final da revisão sistemática da literatura.

Assim, está descrito na Tabela 1, o processo de seleção para a composição da amostra final dos estudos da revisão sistemática.

Os resultados foram extraídos utilizando-se um quadro sinóptico, no qual foram incluídas informações detalhadas de cada estudo, tais como: referência bibliográfica, tipo de estudo, variáveis que caracterizam os sujeitos, limitações metodológicas, evidência dos resultados, aplicabilidade dos resultados, vantagens e desvantagens da intervenção analisada. Tal instrumento foi elaborado, de acordo com as recomendações de outro estudo(11).

Tabela 1- Distribuição dos artigos encontrados e selecionados, Fortaleza-CE, 2013

\begin{tabular}{|c|c|c|c|c|c|c|c|}
\hline Artigos/Base & LILACS & PUBMED & CINAHL & SCOPUS & COCHRANE & $\begin{array}{l}\text { WEB OF } \\
\text { SCIENCE }\end{array}$ & Total \\
\hline Encontrados & 0 & 37 & 14 & 55 & 6 & 8 & 120 \\
\hline Excluídos por não se tratarem de ensaios clínicos & 0 & 24 & 6 & 9 & 2 & 2 & 43 \\
\hline Excluídos por não abordarem a temática & 0 & 7 & 7 & 39 & 1 & 6 & 60 \\
\hline Excluídos por estarem repetidos em outras bases & 0 & 6 & 1 & 7 & 0 & 0 & 14 \\
\hline Selecionados & 0 & 0 & 0 & 0 & 3 & 0 & 3 \\
\hline
\end{tabular}


Por fim, os dados extraídos dos estudos incluídos nesta revisão sistemática foram analisados e apresentados de forma descritiva.

\section{RESULTADOS}

Três ensaios clínicos controlados e randomizados foram incluídos na amostra final da revisão sistemática. Em relação à caracterização dos três estudos selecionados, todos foram publicados a partir do ano de 2005, sendo dois estudos realizados em países europeus ${ }^{(7,16)}$, notadamente no Reino Unido e o outro realizado em um país asiático ${ }^{(17)}$. Segundo a escala de avaliação da qualidade metodológica dos estudos para a composição da revisão sistemática ${ }^{(14)}$, dois obtiveram pontuação três na referida escala $^{(16-17)}$ e um obteve nota quatro ${ }^{(7)}$.

Os resultados dos três estudos selecionados, analisados sob a ótica dos efeitos da sondagem nasogástrica em pacientes com acidente vascular cerebral disfágicos são apresentados no Quadro 2.

Quadro 2 - Distribuição dos estudos selecionados, segundo número de sujeitos por grupo de intervenção, desfechos e resultados principais, Fortaleza-CE, 2013

\begin{tabular}{|c|c|}
\hline \multicolumn{2}{|r|}{ Estudo $1^{(17)}$} \\
\hline Objetivo & $\begin{array}{l}\text { Comparar o estado nutricional e a ocorrência de falha do tratamento entre pacientes com acidente vascular cerebral } \\
\text { e disfagia que receberam a sonda nasogástrica ou a gastrostomia endoscópica percutânea. }\end{array}$ \\
\hline $\begin{array}{l}\text { Intervenção } \\
\text { avaliada }\end{array}$ & $\begin{array}{l}\text { Comparação entre dois métodos de nutrição enteral - sondagem nasogástrica e gastrostomia endoscópica per- } \\
\text { cutânea. }\end{array}$ \\
\hline $\begin{array}{l}\text { Grupos } \\
\text { estudados }\end{array}$ & $\begin{array}{l}22 \text { pacientes com acidente vascular cerebral e disfagia } \\
\text { - } 12 \text { pacientes com sonda nasogástrica } \\
\text { - } 10 \text { pacientes com gastrostomia endoscópica percutânea }\end{array}$ \\
\hline Desfechos & $\begin{array}{l}\text { Estado nutricional: parâmetros antropométricos (prega tricipital e bicipital e circunferência braquial) e marcadores } \\
\text { nutricionais, como nível sérico de albumina. } \\
\text { Falha do tratamento: ocorrência de deslocamento ou bloqueio da sonda alimentar em três ou mais ocasiões. }\end{array}$ \\
\hline $\begin{array}{l}\text { Resultados } \\
\text { Principais }\end{array}$ & $\begin{array}{l}\text { Após quatro semanas de intervenção, os níveis de albumina sérica foram significativamente maiores naqueles que } \\
\text { estavam com a gastrostomia }(p=0,045) \text {. } \\
\text { A ocorrência de falha do tratamento aconteceu em } 50 \% \text { dos pacientes em uso da sonda nasogástrica e nenhum pa- } \\
\text { ciente com gastrostomia endoscópica obteve falha em seu tratamento. Esta diferença foi estatisticamente significativa } \\
\text { entre os dois grupos }(p=0,036) \text {. }\end{array}$ \\
\hline \multicolumn{2}{|r|}{ Estudo $2^{(7)}$} \\
\hline Objetivo & Investigar os efeitos de uma nova técnica de fixação da sonda nasogástrica. \\
\hline $\begin{array}{l}\text { Intervenção } \\
\text { avaliada }\end{array}$ & $\begin{array}{l}\text { Comparação entre dois métodos de fixação da sonda nasogástrica: técnica convencional (uso de um adesivo nasal) } \\
\text { e nova técnica (uma corda com um clipe nasal - AMT Bridle }{ }^{\text {) }} \text {. }\end{array}$ \\
\hline $\begin{array}{l}\text { Grupos } \\
\text { estudados }\end{array}$ & $\begin{array}{l}104 \text { pacientes com acidente vascular cerebral e disfagia } \\
\text { - } 51 \text { pacientes no grupo de intervenção (nova técnica de fixação do tubo) } \\
\text { - } 53 \text { pacientes no grupo controle (técnica convencional) }\end{array}$ \\
\hline Desfechos & $\begin{array}{l}\text { Primário: proporção de alimentos e fluidos administrados por sonda nasogástrica. } \\
\text { Secundários: média do volume de alimentos e fluidos administrados; proporção de pacientes que não receberam } \\
\text { nenhuma alimentação por sonda nasogástrica; administração de fluidos parenterais suplementares; número de in- } \\
\text { tubações nasogástricas; número de Raios-X para checar a posição da sonda nasogástrica; mudanças no peso cor- } \\
\text { poral; falha do tratamento (gastrostomia endoscópica percutânea), precoce ou retirada da alimentação por sonda } \\
\text { nasogástrica); eventos adversos como trauma nasal, infecções pulmonares, diarreia, vômitos, hemorragia gastrointes- } \\
\text { tinal, distúrbios eletrolíticos e a tolerância ao procedimento. } \\
\text { Após três meses da intervenção, os desfechos secundários avaliados foram mortalidade, aumento do tempo de inter- } \\
\text { nação hospitalar, uso de gastrostomia endoscópica percutânea e o Índice de Barthel. }\end{array}$ \\
\hline
\end{tabular}




\begin{tabular}{|c|c|}
\hline $\begin{array}{l}\text { Resultados } \\
\text { Principais }\end{array}$ & $\begin{array}{l}\text { O grupo de intervenção recebeu uma alta proporção de alimentos e fluidos administrados quando comparados ao } \\
\text { grupo controle ( } p=0,002) \text {. } \\
\text { Os pacientes do grupo de intervenção receberam um maior volume de alimentos e fluidos por sonda nasogástrica } \\
\text { (média de } 17 \text { litros versus } 11,4 \text { litros) e necessitaram de menos dias de alimentação suplementar por outras vias } \\
\text { (média de } 3,8 \text { dias versus } 6,1 \text { dias). } \\
\text { Falhas do tratamento foram mais comuns no grupo controle }(40 \%) \text { quando comparado ao grupo de intervenção }(25 \%) \text {. } \\
\text { Sangramento nasal foi mais comum no grupo de intervenção ( } 37 \%) \text { do que no grupo controle }(15 \%) \text {. } \\
\text { Distúrbios eletrolíticos foram mais comuns no grupo controle ( } 58 \% \text { versus } 31 \%) \text {. } \\
\text { Não houve diferença estatisticamente significativa entre os grupos quanto à tolerância ao procedimento }(p=0,09) \text {. } \\
\text { Os resultados da Escala de Barthel foram similarmente ruins entre os grupos (média de } 19 \text { pontos para o grupo de } \\
\text { intervenção e de } 18 \text { pontos para o grupo controle). }\end{array}$ \\
\hline \multicolumn{2}{|r|}{ Estudo $3^{(16)}$} \\
\hline Objetivo & $\begin{array}{l}\text { O objetivo do trabalho foi responder a duas questões de pesquisa: } \\
\text { - A iniciação precoce da alimentação por sonda nasogástrica melhora os resultados dos pacientes com acidente } \\
\text { vascular cerebral e disfagia? } \\
\text { - Os pacientes que se alimentam por gastrostomia endoscópica percutânea obtém melhores resultados quando com- } \\
\text { parado àqueles que utilizam o método de sondagem nasogástrica? }\end{array}$ \\
\hline $\begin{array}{l}\text { Intervenções } \\
\text { avaliadas }\end{array}$ & $\begin{array}{l}\text { Intervenção } 1 \text { - Comparação entre o tempo precoce (até } 7 \text { dias) de iniciar a alimentação por sonda nasogástrica com } \\
\text { o tempo usual (após } 7 \text { dias). } \\
\text { Intervenção } 2 \text { - Comparação entre dois métodos de nutrição enteral - sondagem nasogástrica e gastrostomia per- } \\
\text { cutânea endoscópica }\end{array}$ \\
\hline $\begin{array}{l}\text { Grupos } \\
\text { estudados }\end{array}$ & $\begin{array}{l}\text { Intervenção } 1 \text { - } 859 \text { pacientes (429 iniciaram precocemente a alimentação por sonda nasogástrica e } 430 \text { iniciaram } \\
\text { a alimentação por sonda nasogástrica no tempo usual). } \\
\text { Intervenção } 2 \text { - } 321 \text { pacientes (162 utilizaram a gastrostomia percutânea endoscópica e } 159 \text { utilizaram a sonda } \\
\text { nasogástrica). }\end{array}$ \\
\hline Desfechos & $\begin{array}{l}\text { Primário: a escala de Rankin Modificada e a qualidade de vida com a Escala EUROQol, risco de morte e de prognós- } \\
\text { tico ruim (complicações) e tempo de internação hospitalar. }\end{array}$ \\
\hline $\begin{array}{l}\text { Resultados } \\
\text { Principais }\end{array}$ & $\begin{array}{l}\text { A incidência de hemorragia gastrointestinal foi maior naqueles com sonda nasogástrica }(p=0,05) \text {. } \\
\text { Não houve diferença estatisticamente significativa nos resultados (morte e piora do estado de saúde em seis meses) } \\
\text { dos pacientes que iniciaram precocemente a alimentação por sonda nasogástrica }(p=0,09 \text { e } p=0,07) \text { e daqueles que } \\
\text { iniciaram no tempo padrão }(p=0,09 \text { e } p=0,06) \text {. } \\
\text { Na comparação da Intervenção } 2 \text { (sondagem nasogástrica versus gastrostomia) houve diferença absoluta no risco de } \\
\text { morte ou de prognóstico ruim em favor da sondagem nasogástrica ( } p=0,04) \text {. } \\
\text { Após três meses, os níveis de albumina sérica foram significativamente maiores naqueles que estavam com a gas- } \\
\text { trostomia ( }(p=0,03 \text { ). } \\
\text { Em relação à Escala de Rankin Modificada, a maioria dos pacientes avaliados em todos os grupos tinham a nota } 5 \\
\text { (incapacidade severa). Não houve diferença estatisticamente significativa entre os grupos (alimentação precoce por } \\
\text { sonda }-p=0,06 \text { e alimentação no tempo padrão }-p=0,08 \text { ). }\end{array}$ \\
\hline
\end{tabular}

Os resultados dos estudos selecionados, que buscaram comparar os métodos de alimentação enteral por sondagem nasogástrica e gastrostomia endoscópica percutânea em pacientes com acidente vascular cerebral e disfagia, apontaram diferentes desfechos, entre os quais se destacam: aumento do nível sérico de albumina (gastrostomia), prognóstico ruim e risco de morte (gastrostomia), aumento das falhas no tratamento devido bloqueio, deslocamento e reinserção da sonda (sonda nasogástrica) e aumento da incidência de hemorragia gastrointestinal (sonda nasogástrica) ${ }^{(16-17)}$.
Em relação aos resultados encontrados sobre o melhor método de fixação da sonda nasogástrica, pode ser observado que os pacientes que utilizaram a nova técnica de fixação (corda com um clipe nasal - AMT Bridle ${ }^{\circ}$ ) obtiveram menores taxas de falhas no tratamento e de distúrbios eletrolíticos e aumento do volume de alimentos e fluidos administrados por sonda nasogástrica. Entretanto, não houve diferença quanto aos escores da Escala de Barthel e tolerância ao procedimento ${ }^{(7)}$.

Por fim, a comparação entre o tempo precoce de início de uma dieta por sonda nasogástrica (até 7 dias) e o tempo usual 
(acima de 7 dias) não apontou diferenças entre os grupos quanto à presença de complicações e melhora dos índices da Escala de Rankin Modificada ${ }^{(16)}$.

\section{DISCUSSÃO}

Em relação à incidência de disfagia em pacientes com acidente vascular cerebral, vários estudos referem que esta ocorre em 30 a 50\% destes pacientes. Cerca de $40 \%$ destes desenvolvem a aspiração respiratória e 37\% dos que aspiram evoluem para a pneumonia aspirativa ${ }^{(5,18)}$. Quanto ao tempo para o desenvolvimento da disfagia, autores ${ }^{(19-20)}$ relatam que estaria clinicamente presente em 42 a $67 \%$ dos pacientes nos primeiros três dias da ocorrência do acidente vascular cerebral.

Assim, em virtude da incidência de disfagia em pacientes com acidente vascular cerebral, do risco elevado de aspiração respiratória e das complicações em termos de morbidade, mortalidade e custos, a identificação da melhor terapia para nutrição enteral se faz necessária.

Pesquisa desenvolvida anteriormente ${ }^{(21)}$ constatou que o efetivo e precoce tratamento da disfagia reduziu a incidência da pneumonia aspirativa e que cuidados padronizados da equipe de saúde quanto à alimentação enteral por sonda nasogástrica reduziu o risco de aspiração nos pacientes com acidente vascular cerebral e disfagia.

Destarte, levanta-se a necessidade da elaboração e testagem de protocolos clínicos de terapia nutricional por meio da sonda nasogástrica em pacientes com acidente vascular cerebral disfágicos, incluindo uma avaliação precoce do risco de aspiração e do estado da deglutição.

Um dos ensaios clínicos encontrados neste estudo(16) apontou que não houve diferença estatisticamente significativa entre os resultados dos pacientes que iniciaram precocemente a alimentação por sonda nasogástrica (anterior a sete dias) quando comparados àqueles que iniciaram no tempo padrão (após sete dias) e recomendaram a iniciação precoce da alimentação enteral por sonda nasogástrica nos primeiros dias de admissão dos pacientes com acidente vascular cerebral e disfagia.

Outra intervenção testada ${ }^{(7)}$ foi a adoção de um clipe nasal (AMT Bridle ) que facilita a fixação da sonda nasogástrica e que promoveu a diminuição de falhas no tratamento nutricional, como o deslocamento e reinserção da sonda nasogástrica.

Tais estudos nos permitem inferir que as falhas do tratamento da sonda nasogástrica geralmente envolvem falhas técnicas na inserção, fixação e na manutenção da sonda nasogástrica em ambiente hospitalar e domiciliar. Procedimentos corretos na instalação da sonda e na administração da dieta são de vital importância para evitar as complicações e fazer com que o paciente receba os benefícios da terapia nutricional.

Os dois ensaios clínicos que compararam dois métodos de nutrição enteral - sonda nasogástrica e gastrostomia endoscópica percutânea ${ }^{(16-17)}$ concluíram que os níveis de albumina sérica foram maiores naqueles pacientes em uso de gastrostomia.

A subnutrição é um problema que compromete a evolução dos pacientes hospitalizados. Dessa forma, a avaliação nutricional, com destaque para a obtenção dos níveis séricos de albumina, se faz importante para o acompanhamento dos pacientes com acidente vascular cerebral e disfagia ${ }^{(16)}$. É oportuno salientar que, provavelmente o aumento sérico de albumina (indicador de melhora do estado nutricional) não se deve apenas ao método de terapia nutricional - gastrostomia, mas ao fato da sonda nasogástrica ser indicada na fase aguda da doença, onde acontece maior depleção proteica e calórica $^{(19)}$.

Em meta-análise recente ${ }^{(22)}$, a gastrostomia endoscópica percutânea esteve associada à menor incidência de interrupções na alimentação, obstrução ou perda do posicionamento da sonda e não aderência ao tratamento quando comparada com a sondagem nasogástrica, no entanto, não houve diferença significativa em relação ao número de complicações, taxa de mortalidade e incidência de pneumonia.

Um outro estudo ${ }^{(23)}$ apontou que a gastrostomia endoscópica percutânea é o método de escolha para nutrição enteral prolongada em pacientes com diversos distúrbios, incluindo os que tem acidente vascular cerebral. Quando comparado com outros métodos de nutrição enteral, como a alimentação por sonda nasoentérica, a gastrostomia apresenta vantagens em termos de conforto para o paciente, além de menores taxas de sangramento local, obstrução do conduto de alimentação e deslocamento do tubo (falhas do tratamento). Entretanto, não impede o refluxo gastroesofágico ou a aspiração do conteúdo gástrico.

Ademais, é oportuno considerar também que, apesar dos benefícios apontados nos ensaios clínicos citados, a gastrostomia é um procedimento mais invasivo, mais caro e necessita de profissionais especializados para a sua inserção, em relação ao procedimento de sondagem nasogástrica.

\section{CONCLUSÃO}

A partir dos resultados obtidos nesta revisão sistemática, foram encontradas as seguintes evidências sobre os efeitos da sondagem gástrica em pacientes com acidente vascular cerebral e disfágicos:

- A sondagem nasogástrica deve ser adotada precocemente como um método de alimentação enteral.

- As falhas do tratamento (bloqueio, deslocamento da sonda e reinserção da sonda) são mais comuns naqueles que utilizam a sonda nasogástrica como método de alimentação enteral.

- Há riscos e benefícios da gastrostomia endoscópica percutânea quando comparada à sondagem nasogástrica.

- Os resultados relacionados à melhora do estado funcional do paciente obtido por meio de escalas como a Rankin Modificada e a de Barthel foram semelhantes entre os pacientes, independente do método de terapia nutricional empregado.

Enfatiza-se novamente a necessidade de realização de outros estudos que abordem a elaboração e testagem de protocolos clínicos sobre a técnica correta de inserção e manutenção da alimentação enteral por sondagem nasogástrica direcionados à equipe de enfermagem, aos pacientes e cuidadores. 


\section{REFERÊNCIAS}

1. Cavalcante TF, Araújo TL, Moreira RP, Guedes NG, Lopes MVO, Silva VM. Clinical validation of the nursing diagnosis Risk for aspiration among patients who experencied a cerebrovascular accident. Rev Latinoam Enferm [Internet]. 2013 [cited 2013 February 2];21(Spec No):250-8. Available from: http://www.scielo.br/scielo.php?pid=S0104$11692013000700031 \&$ script $=$ sci_abstract

2. Foley N, Teasell R, Salter K, Kruger E, Martino R. Dysphagia treatment post stroke: a systematic review of randomized controlled trials. Age Ageing. 2008;37(3):258-64.

3. Masiero S, Pierobon R, Previato C, Gomiero E. Pneumonia in stroke patients with oropharyngeal dysphagia: a sixmonth follow-up study. Neurol Sci. 2008;29(3):139-45.

4. Need H. Dysphagia Following Stroke. Int J Lang Comm Disord. 2010;45(6):710-1.

5. Campbell-Taylor IC. Oropharyngeal dysphagia in longterm care: misperceptions of treatment efficacy. J Am Med Dir Assoc. 2008;9(7):523-31.

6. Morris H. Dysphagia: implications of stroke on swallowing. Nurs Resid Care. 2008;10(8):378-81.

7. Beavan J, Conroy SP, Harwood R, Gladman JRF, LeonardiBee J, Sach T, et al. Does looped nasogastric tube feeding improve nutritional delivery for patients with dysphagia after acute stroke? A randomized controlled trial. Age Ageing. 2010;39(5):624-30.

8. Oliveira SM, Burgos MGPA, Santos EMC, Prado LVS, Petribú MMV, Bomfim FMTS. Gastrointestinal complications and protein-calorie adequacy in intensive care unit enteral nutrition patients. Rev Bras Ter Intensive [Internet]. 2010 [cited 2014 July 12];22(3):270-3. Available from: http://www.scielo.br/scielo.php?pid=S0103$507 X 2010000300009 \&$ script $=$ sci_arttext\&tlng $=$ en

9. Summers D, Leonard A, Wentworth D, Saver JL, Simpson J, Spilker JA, et al. Comprehensive overview of nursing and interdisciplinary care of to the acute ischemic stroke patient. Stroke. 2009;40:2911-44.

10. Cullum N, Ciliska D, Haynes R B, Marks S. Enfermagem Baseada em Evidências: uma introdução. Porto Alegre (BR): Artmed; 2010.

11. De-la-Torre-Ugarte-Guanilo MC, Takahashi RF, Bertolozzi MR. Systematic review: general notions. Rev Esc Enferm USP [Internet]. 2011 [cited 2012 December 02];45(5):1260-6. Available from: http://www.scielo.br/scielo.php?script $=$ sci arttext\&pid = S0080-62342011000500033\&lng = en\&nrm $=\mathrm{iso \& t \operatorname {lng }}=\mathrm{en}$

12. Proveda VB, Clark AM, Galvão CM. A systematic review on the effectiveness of prewarming to prevent perioperative hypothermia. J Clin Nurs. 2013;22(7-8):906-18.

13. Santos CMC, Pimenta CAM, Nobre MRC. The PICO Strategy for the research question construction and evidence search. Rev Latinoam Enferm. 2007;15(3):508-11.

14. Jadad AR, Moore RA, Carroll D, Jenkinson C, Reynolds DJ, Gavaghan DJ, et al. Assessing the quality of reports of randomized clinical trials: is blinding necessary? Control Clin Trials [Internet]. 1996 [cited 2012 October 11];17(1):1-12. Available from: http://www.ncbi.nlm.nih. gov/pubmed/8721797

15. Slater AE. Randomized controlled trials. In: Jadad AR. The Handbook of clinical trials and other research. London (ENG): BMJ Books; 1998.

16. Clarke J, Granswick G, Dennis MS, Flaig R, Fraser A, Grant $\mathrm{S}$, et al. Effect of timing and method of enteral tube feeding for dysphagic stroke patients (FOOD): a multicenter randomized controlled trial. Lancet [Internet]. 2005 [cited 2012 May 23];365(9461):764-72. Available from: http:// www.ncbi.nlm.nih.gov/pubmed/15733717

17. Hamidon BB, Abdullah SA, Zawawi MF, Sukumar N, Aminuddin A, Raymond AA. A prospective comparison of percutaneous endoscopic gastrostomy and nasogastric tube feeding in patients with acute dysphagic stroke. Med J Malaysia [Internet]. 2006 [cited 2012 May 23];61(1):5966. Available from: http://www.ncbi.nlm.nih.gov/pubmed/ 16708735

18. Smith Hammond CA, Goldstein LB, Horner RD, Ying J, Gray L, Gonzalez-Rothi L, et al. Predicting aspiration in patients with ischemic stroke: comparison of clinical signs and aerodynamic measures of voluntary cough. Chest [Internet]. 2009 [cited 2012 May 02];135(3):769-77. Available from: http://www.ncbi.nlm.nih.gov/pubmed/19017886

19. Serra-Prat M, Palomera M, Gomez C, Sar-Shalom D, Saiz A, Montoya JG, et al. Oropharyngeal dysphagia as a risk factor for malnutrition and lower respiratory tract infection in independently living older persons: a populationbased prospective study. Age Ageing. 2012;41(3):376-81.

20. Martino R, Silver F, Teasell R, Bayley M, Nicholson G, Streiner DL, et al. The Toronto Bedside Swallowing Screening Test (TOR-BSST): development and validation of a dysphagia screening tool for patients with stroke. Stroke. 2009;40(2):555-61.

21. Garcia JM, Chambers E, Clark M, Helverson J, Matta Z. Quality of care issues for dysphagia: modifications involving oral fluids. J Clin Nurs [Internet]. 2010 [cited 2012 May 15];19(11):1618-24. Available from: http://www. ncbi.nlm.nih.gov/pubmed/20384670

22. Gomes CA Jr, Lustosa SA, Matos D, Andriolo RB, Waisberg DR, Waisberg J. Percutaneous endoscopic gastrostomy versus nasogastric tube feeding for adults with swallowing disturbances. Cochrane Database Syst Rev [Internet]. 2010 [cited 2012 May 23];10(11):CD008096. Available from: http://www.ncbi.nlm.nih.gov/pubmed/21069702

23. Santos JS, Kemp R, Sankarankutty AK, Salgado Júnior W, Tirapelli LF, Silva Júnior OC. Gastrostomia e jejunostomia: aspectos da evolução técnica e da ampliação das indicações. Medicina (Ribeirão Preto) [Internet]. 2011 [acesso em 25 de abril de 2013];44(1):39-50. Disponível em: http://revista.fmrp.usp.br/2011/vol44n1/Simp4 Gastrostomia_e_jejunostomia\%20atual.pdf 